\title{
Neurophotonics
}

Neurophotonics.SPIEDigitalLibrary.org

\section{Larry Cohen-50 ways to DYE your science}

\author{
Timo Strünker \\ U. Benjamin Kaupp
}

\section{SPIE.}




\section{Larry Cohen-50 ways to DYE your science}

Timo Strünker and U. Benjamin Kaupp*

Center of Advanced European Studies and Research, Department of Molecular Sensory Systems, Ludwig-Erhard-Allee 2, 53175 Bonn, Germany

Larry Cohen has been a great inspiration for our work; the indicators Arsenazo III and di-8-ANNEPS, introduced by Larry and his colleagues to biology, ${ }^{1,2}$ were crucial for the elucidation of photo- and chemotransduction pathways in photoreceptors and sperm cells, respectively. During the heydays of research on signaling in retinal photoreceptors in the 1980s, central questions concerned (1) the identity of the cellular messenger transducing the absorption of light into an electrical signal and (2) the nature of the ion channels gated by that messenger. For good reasons, both $\mathrm{Ca}^{2+}$ and cGMP had been proposed to carry the signal from the visual pigment rhodopsin in the disk membrane across the cytosol to the ion channels in the plasma membrane. In 1985, in one fell swoop, a series of papers identified in rod photoreceptors of amphibians and cow a conductance, which is directly gated by cGMP without involving phosphorylation by protein kinase $\mathrm{G}^{3,4,5} \mathrm{We}$ and others discovered a cGMP-induced $\mathrm{Ca}^{2+}$ efflux from isolated $\mathrm{Ca}^{2+}$-filled disks, ${ }^{4,6}$ suggesting that the cGMPgated channel is also localized in the disk membrane. The release of $\mathrm{Ca}^{2+}$ from isolated disks was detected by the metallochromic $\mathrm{Ca}^{2+}$-indicator dye Arsenazo III. This dye, for the first time, was used by Brown et al. ${ }^{1}$ to measure minute $\mathrm{Ca}^{2+}$ changes in the squid giant axon evoked by changes in membrane voltage. Recordings from excised membrane patches or truncated rod outer segments revealed that the cGMP-gated channel is, in fact, located in the plasma membrane..$^{3,5}$ It turned out that robust cytoskeletal filaments connect plasma and disk membranes; ${ }^{7}$ therefore, during permeabilization of photoreceptors and purification of their membrane fractions, plasma and disk membranes partially fuse. This process is prevented by mild trypsinization of cytoskeletal elements. ${ }^{8}$ Although the presence of the cGMP-gated channel in disks was a consequence of the isolation procedure, it allowed for the channel's molecular identification, purification, and cloning of the gene. We characterized the cGMP-gated channel by functional reconstitution into artificial liposomes. ${ }^{9,10}$ Membrane proteins of rod outer segments were solubilized and separated by affinity and size-exclusion chromatography; protein fractions eluting from the column were reconstituted into liposomes and tested for channel activity using the Arsenazo III-based $\mathrm{Ca}^{2+}$ flux assay. ${ }^{9}$ This assay also allowed identifying the channel's accessory $240 \mathrm{kD} \beta$-subunit ${ }^{11}$ and the first $\mathrm{Na}^{+} / \mathrm{Ca}^{2+}$ exchanger protein. ${ }^{12}$ Finally, partial aminoacid information derived from the purified $63 \mathrm{kD}$ pore-forming $\alpha$-subunit and the $\beta$-subunit paved the way to clone the genes of the CNG channel subunits and their functional expression in heterologous cell systems. ${ }^{13,14}$

It came as a surprise that relatives of these CNG channels are essential components of chemotactic signaling in sperm. ${ }^{15,16,17}$ Stimulation of sea urchin sperm with chemoattractants, short peptides, causes a hyperpolarization
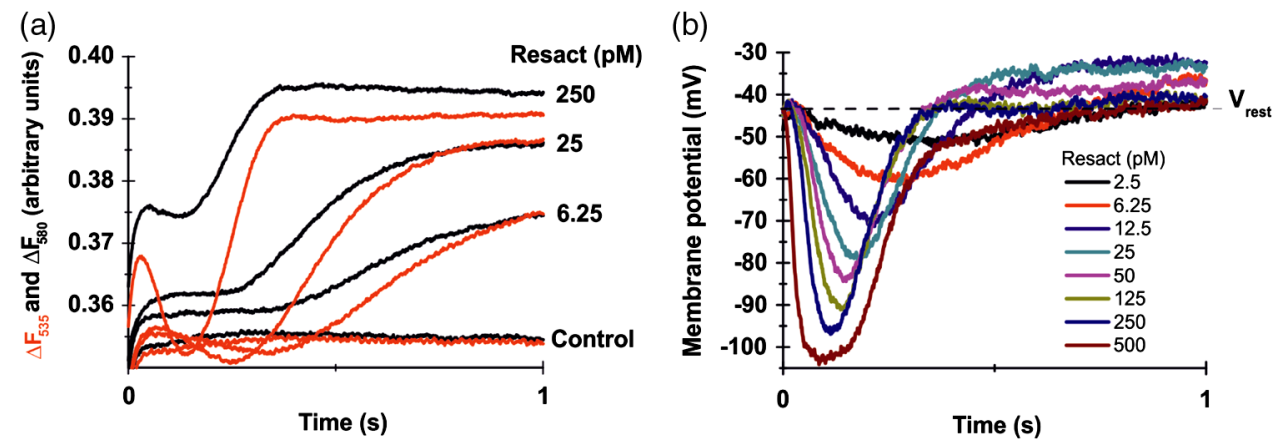

Fig. 1 Quantitative fluorescence optical recordings of chemoattractant-induced voltage responses in sea urchin sperm in suspension stained with di-8-ANNEPS were mixed in a stopped-flow device with the chemoattractant peptide "resact." The dye was excited at $480 \mathrm{~nm}$. (a) Resact-induced changes in di8-ANNEPS fluorescence emission recorded simultaneously at $535 \mathrm{~nm}$ (red) and $580 \mathrm{~nm}$ (black) after stimulation of sperm at $t=0$. Resact evoked opposite fluorescence changes at 535 and $580 \mathrm{~nm}$ that were superposed on a persistent increase of fluorescence at both wavelengths. (b) By taking the ratio $R=F 580 / F 535$, the steady component of the fluorescence signal is eliminated, unravelling that resact evokes a pulse-like, transient hyperpolarization, followed (at resact $\geq 50 \mathrm{pM}$ ) by a slow persistent depolarization. Changes in $R$ were calibrated to yield changes in $V_{m}$.

*Address all correspondence to U. Benjamin Kaupp, E-mail: U.B.Kaupp@ caesar.de 
followed by a transient $\mathrm{Ca}^{2+}$ response. Electrical recording from mouse and human sperm had been established, ${ }^{18,19,20}$ whereas direct probing of electrical events in sea urchin sperm by the patch-clamp technique was unsuccessful. Instead, fluorescent potentiometric probes had been employed to record changes in membrane voltage. Owing to their "Nernstian mechanism" of voltage sensing, the dyes initially used for sperm studies exhibit slow response times and, moreover, are prone to solvatochromic artefacts. ${ }^{21}$ We sought the advice of Larry Cohen, who recommended di-8-ANEPPS, a dye that exclusively intercalates into the plasma membrane and exhibits a rapid response at the millisecond time scale. Its electrochromic mechanism of voltage sensing allows ratiometric measurement of $\Delta V_{m}$ and, thereby, overcomes potential artefacts resulting from unspecific solvatochromic effects. ${ }^{17}$ The necessity of ratiometric measurements is illustrated in Fig. 1. Di-8-ANEPPS faithfully and, most importantly, quantitatively reports $\Delta V_{m}$ in intact, freely moving sperm; optoelectrical recordings from sperm revealed that stimulation with a single chemoattractant molecule evokes a $\Delta V_{m}$ of about $2.5 \mathrm{mV},{ }^{17}$ similar to the single-photon response of rod photoreceptors. The dye also allows using flash photolysis of caged compounds that exhibit absorption spectra around $400 \mathrm{~nm} .^{15,17}$ Competing dyes like the Annine collection of potentiometric probes require excitation wavelengths $<500 \mathrm{~nm}$, which interferes with the uncaging of cyclic nucleotides at ca. $410 \mathrm{~nm}$, using coumaryl-based caging groups. ${ }^{22-24}$ In conclusion, up to this day, di-8-ANEPPS has been key for delineating the sequence of signaling events during chemotaxis of sea urchin sperm and for identifying the underlying signaling molecules. ${ }^{25,26}$

Apart from our love for dyes and photonics, we share an appreciation for sweet German Spätlese or Auslese Rieslings. We promise to continue to import them to the US. For more than 10 years, Larry has been generously hosting Benjamin Kaupp and his wife, Sigi, in his Woods Hole home during the summer. We do not want to miss the great conversations and jokes at the dinner table, the unique blend of food, wine and people, and, most importantly, Larry's tireless hospitality. He is a great scholar and friend!

\section{References}

1. J. E. Brown et al., "Rapid changes of intracellular free calcium concentration: detection by metallochromic indicator dyes in squid giant axon," Biophys. J. 15, 1155-1160 (1975).

2. E. Fluhler et al., "Spectra, membrane binding, and potentiometric responses of new charge shift probes," Biochemistry 24(21), 57495555 (1985).

3. E. E. Fesenko, S. S. Kolesnikov, and A. L. Lyubarsky, "Induction by cyclic GMP of cationic conductance in plasma membrane of retinal rod outer segment," Nature 313, 310-313 (1985).

4. K.-W. Koch and U. B. Kaupp, "Cyclic GMP directly regulates a cation conductance in membranes of bovine rods by a cooperative mechanism," J. Biol. Chem. 260, 6788-6800 (1985).
5. K.-W. Yau and K. Nakatani, "Light-suppressible, cyclic GMP-sensitive conductance in the plasma membrane of a truncated rod outer segment," Nature 317, 252-255 (1985).

6. A. Caretta and A. Cavaggioni, "Fast ionic flux activated by cyclic GMP in the membrane of cattle rod outer segments," Eur. J. Biochem. 132, 1-8 (1983).

7. D. J. Roof and J. E. Heuser, "Surfaces of rod photoreceptor disk membranes: Integral membrane components," J. Cell. Biol. 95, 487-500 (1982).

8. N. J. Cook et al., "The cGMP-gated channel of bovine rod photoreceptors is localized exclusively in the plasma membrane," J. Biol. Chem. 264(12), 6996-6999 (1989).

9. N. J. Cook, W. Hanke, and U. B. Kaupp, "Identification, purification, and functional reconstitution of the cyclic GMP-dependent channel from rod photoreceptors," Proc. Natl. Acad. Sci. USA 84, 585-589 (1987).

10. N. J. Cook et al., "Solubilization and functional reconstitution of the cGMP-dependent cation channel from bovine rod outer segments," J. Biol. Chem. 261(36), 17033-17039 (1986).

11. L. L. Molday et al., "The cGMP-gated cation channel of bovine rod photoreceptor cells is associated with a 240-kDa protein exhibiting immunochemical cross-reactivity with spectrin," J. Biol. Chem. 265 (30), 18690-18695 (1990).

12. N. J. Cook and U. B. Kaupp, "Solubilization, purification, and reconstitution of the sodium-calcium exchanger from bovine retinal rod outer segments," J. Biol. Chem. 263(23), 11382-11388 (1988).

13. U. B. Kaupp et al., "Primary structure and functional expression from complementary DNA of the rod photoreceptor cyclic GMP-gated channel," Nature 342, 762-766 (1989).

14. H. G. Körschen et al., "A $240 \mathrm{kDa}$ protein represents the complete $\beta$ subunit of the cyclic nucleotide-gated channel from rod photoreceptor," Neuron 15, 627-636 (1995).

15. W. Bönigk et al., "An atypical CNG channel activated by a single cGMP molecule controls sperm chemotaxis," Sci. Signal 2, ra68 (2009).

16. R. Gauss, R. Seifert, and U. B. Kaupp, "Molecular identification of a hyperpolarization-activated channel in sea urchin sperm," Nature 393, 583-587 (1998).

17. T. Strünker et al., "A K+ -selective cGMP-gated ion channel controls chemosensation of sperm," Nat. Cell. Biol. 8, 1149-1154 (2006).

18. Y. Kirichok, B. Navarro, and D. E. Clapham, "Whole-cell patch-clamp measurements of spermatozoa reveal an alkaline-activated $\mathrm{Ca}^{2+}$ channel," Nature 439, 737-740 (2006).

19. P. V. Lishko, I. L. Botchkina, and Y. Kirichok, "Progesterone activates the principal $\mathrm{Ca}^{2+}$ channel of human sperm," Nature 471, 387-391 (2011).

20. T. Strünker et al., "The CatSper channel mediates progesterone-induced Ca2+ influx in human sperm," Nature 471, 382-386 (2011).

21. J. Plasek and K. Sigler, "Slow fluorescent indicators of membrane potential: a survey of different approaches to probe response analysis," J. Photochem. Photobiol. B 33, 101-124 (1996).

22. V. Hagen et al., "Highly efficient and ultrafast phototriggers for cAMP and cGMP by using long-wavelength UV/Vis-activation," Angew. Chem. Int. Ed. 40, 1046-1048 (2001).

23. V. Hagen et al., "Fluorescence spectroscopic quantification of the release of cyclic nucleotides from photocleavable [Bis(carboxymethoxy)coumarin-4-yl]methyl esters inside cells," Angew. Chem. Int. Ed. 41, 3625-3628 (2002).

24. V. Hagen et al., "[7-(Dialkylamino)coumarin-4-yl]methyl-caged compounds as ultrafast and effective long-wavelength phototriggers of 8bromo-substituted cyclic nucleotides," Chem. Biol. Chem. 4(5), 434442 (2003).

25. L. Alvarez et al., "The computational sperm cell," Trends Cell. Biol. 24, 198-207 (2014).

26. U. B. Kaupp, "100 years of sperm chemotaxis,” J. Gen. Physiol. 140, 583-586 (2012). 